\title{
NUMERICAL SIMULATION OF AIRFLOW OVER POTENTIAL TELESCOPE SITES
}

\author{
DAVID S. DE YOUNG
}

Kitt Peak National Observatory, National Optical Astronomy Observatories,' 950 N. Cherry Avenue, P.O. Box 26732, Tucson,

Arizona 85726-6732

Electronic mail: deyoung@/noao.edu

RICHARD D. CHARLES

San Diego Supercomputer Center and Department of Applied Mechanics and Engineering Sciences,

University of California at San Diego, La Jolla, California 92093

Electronic mail: charies@sdsc.edu

Received 1995 June 20; revised 1995 August 31

\begin{abstract}
Numerical simulation can provide a valuable tool in evaluating the characteristics of possible sites for new telescopes. This technique can provide accurate information about topographically induced atmospheric disturbances and turbulent boundary layers occurring in the high Reynolds number airflows over such sites. Results are given here for simulations of airflow at the two Gemini 8-m telescope sites on Mauna Kea and Cerro Pachon. These calculations permitted an informed choice to be made between two sites on Cerro Pachon; determined that upwind topographic features did not have a significant influence at the Mauna Kea site; and confirmed that the proposed height of the telescope pier would place the primary mirror above the turbulent boundary layer under the majority of wind directions and speeds. @ 1995 American Astronomical Societv.
\end{abstract}

\section{INTRODUCTION}

Recent advances in large-scale mirror design, active mirror control technology, and telescope and enclosure design and control have made possible the design and construction of $10 \mathrm{~m}$ class telescopes with unprecedented performance (e.g., Stepp 1994). When coupled with state-of-theart detectors and instrumentation, these telescopes will begin a new era in our understanding of the universe around us. In order to realize the full potential of these new telescopes it is essential that they be located at the best possible sites. In particular, the technical advances in mirror and telescope design and control can all be undone if the site does not possess seeing characteristics which are on average as good as or better than the image quality that can be delivered by the telescope.

In the past, information on the seeing quality of a given site has sometimes been available in the form of quantitative measurements, but more often it has been anecdotal in nature. (Such anecdotal information, when delivered by experienced observers, has often proved to be very reliable.) Investigation of the seeing qualities of a given proposed telescope site can be carried out by measurements over a period sufficiently long to provide data under a variety of conditions; 
however, if several sites are being considered or if an entire mountain top is being investigated, then such measurements can be prohibitive in terms of time and cost. In this case a useful tool has recently become available which can serve as a valuable supplement to on-site measurements, namely the use of computational fluid dynamics (CFD) to carry out numerical simulations of conditions at a variety of sites.

Such simulations, if carried out in three dimensions with high resolution and accurate topographical representation of the site, can provide valuable data for site selection. These calculations can provide information on the thickness and intensity of turbulent boundary layers over the entire region for a wide variety of wind speeds and directions; they can determine the effects of various topographical features near a proposed site; and they can provide insight into the effects of temperature gradients at the site. All of these can degrade the seeing if their effects intercept the light path to the mirror because they serve to mix parcels of air at different temperatures and produce variations in the refractive index along the light path. Numerical simulations can also provide greater accuracy than water tunnel tests because the latter cannot provide flows with Reynolds numbers as high as those actually present on the site (Wong \& Forbes 1991). Such lower Reynolds number flows are unrealistically viscous and do not accurately predict the behavior of turbulent boundary layers generated by airflow over irregular terrain.

The present set of simulations was carried out in conjunction with possible site locations for the two $8 \mathrm{~m}$ telescopes of the Gemini project (Mountain et al. 1994). One of these telescopes will be located on Mauna Kea in Hawaii and the other on Cerro Pachon in Chile. The calculations for the northern and southern hemisphere sites had slightly different objectives. For the Mauna Kea site two specific questions were posed: first, what effects do prominent topographical features in the summit region have at the proposed site under a variety of wind conditions; and second, what is the optimum height of the telescope support pier so that the light path to the mirror will be above the turbulent boundary layer during the most common wind conditions? The second question is important not only for its impact on the structural design of the telescope but also because of the dramatic increase in construction cost as the height of the support pier and telescope enclosure is raised. These two questions were addressed by two separate sets of simulations on two different scales, as will be described in more detail in Sec. 2. For the Chilean telescope the issue was the choice of one of two possible sites. This choice was to be determined not only by the seeing quality at the two sites but also by other factors such as ease of access and its resulting impact on overall construction costs. In this paper Sec. 2 provides a more detailed description of the specific problems addressed by the simulations, together with an account of the boundary conditions and the method of calculation. The results of these calculations are given in Sec. 3, and these are discussed further in Sec. 4.

FlG. 1. Topographic map of the area covered by the large-scale Mauna Kea simulations. The extent is approximately one mile north-south by 1 2/3 miles east-west. The small rectangle across the summit ridge shows the approximate area covered by the high-resolution calculations. The telescope site is on the summit ridge at the north-south center of this rectangle. The contour interval is 40' except around the summit ridge, where the contour interval is 20'. The contour labeled "A:' is at an altitude of 13 240', with contours rising to the north at that point. North is at the top. 


\section{INITIAL CONDITIONS AND METHOD OF CALCULATION}

\subsection{Mauna Kea}

As mentioned in Sec. 1, the Mauna Kea simulations had two objectives: to determine the effects of large scale topographic features on the airflow over the proposed site, and to provide estimates of the thickness of the turbulent boundary layer at the proposed site under a variety of conditions. The first objective was addressed by considering the flow over a relatively large region of the summit area. This region is shown in Fig. 1 and includes an area roughly one mile $(1.6 \mathrm{~km})$ in extent north-south by $12 / 3$ miles $(2.7 \mathrm{~km})$ in extent cast-west. The proposed site on the summit ridge is noted in the caption to Fig. 1. This particular area was chosen because the prevailing winds in clear weather on Mauna Kea are predominantly from the east or west (Raybould et al. 1994). The rectangle was chosen to extend more to the west than to the east due to greater concern about the effects of the small peaks lying west of the site. These features could produce complex turbulent wakes over the site during a west wind, whereas the more smoothly varying terrain to the east, with its rapid drop to lower elevations, was felt to be of less concern.

FIG. 2. Gridded surface of the area shown in Fig. 1 as used in the calculations. The view angle is looking toward the northeast, and the volume above the surface included in the simulations is shown outlined. The proper perspective is gained by noting that the gridded surface forms the base of the volume shown.

The topographic data for both Mauna Kea and Cerro Pachon were not originally available in the regularly spaced rectilinear form required by the computational procedure, and special software had to be developed to convert the data to this form. Figure 2 shows one view of the Mauna Kea summit region in its gridded form as used for the large scale airflow simulations. Free stream wind speeds of 2, 5, 10, and $20 \mathrm{~m} / \mathrm{s}$ from both east and west were simulated in this set of calculations. These wind speeds span the observed range of wind data on Mauna Kea during clear weather (Raybould et al. 1994). The air density was set at $7.9 \times 10^{-4} \mathrm{~g} \mathrm{~cm}^{-3}$, which is the appropriate value for an elevation of 14000' (4267m) MSL under standard atmospheric conditions.

Examination of the nature of the boundary layer required a higher spatial resolution than that available in the large scale calculation, and hence a detailed simulation of the immediate area around the site was made in a second set of calculations. The area covered by this set measured approximately 1000 ' by 2000 ' $(305 \mathrm{~m} \times 1010 \mathrm{~m})$. This series of calculations included a rough approximation of the telescope enclosure in the form of a right circular cylinder of radius and height equal to that of the proposed $8 \mathrm{~m}$ telescope enclosure. This structure was included in order to accurately simulate the nature of the turbulent boundary layer in the immediate vicinity of the telescope. One view of the resulting grid used in this set of simulations is shown in Fig. 3, where features such as the summit access road on the western flank of the summit ridge can be seen.

\subsection{Cerro Pachon}


The object of this simulation was to compare the airflow over two possible sites on the mountain top, and to do this accurately required a single very large scale simulation volume which included both sites and the highly variable topology of the summit area. Only in this manner could the complex interactions of the airflow at the two sites be reliably evaluated. The area covered by the simulations measured $3 \mathrm{~km}$ by $3 \mathrm{~km}$, and the topography is shown in Fig. 4, together with the proposed site locations. The prevailing winds are from the north, and a particular concern was the possibility of turbulent flow over the primary site induced by upwelling air from the upstream trough in the mountain face during northerly winds. Figure 5 shows the grid of the mountain surface used in the simulations. A $10 \mathrm{~m} / \mathrm{s}$ wind from the north was used here; this speed and direction matched meteorological data as being the most common condition.

FIG. 3. Gridded surface of the high-resolution Mauna Kea summit region as used in the calculations. The proposed telescope enclosure is shown by the cylindrical shape; view is looking toward the northeast.

FIG. 4. Topographic map of the $3 \mathrm{~km} \times 3 \mathrm{~km}$ area of Cerro Pachon used in the simulations. North is at the top, and the contour interval is $5 \mathrm{~m}$. The proposed site locations are shown by $X$ 's, with the eastern site designated as the primary site.

FIG. 5. Grid of the surface of Cerro Pachon as used in the calculations. The view is looking toward the southwest.

\subsection{Method of Calculation}

The fluid dynamic calculations are fully three dimensional, time independent, and incompressible. The general questions posed here are concerned with average or most common airflow conditions at the various sites; hence steady state calculations are appropriate. For the wind speeds considered here, compressibility is not a factor. As mentioned in Sec. 1, these calculations accurately treat flows at the correct Reynolds number $(\mathrm{Re})$ of several tens of millions, where $\operatorname{Re}=U L / v$. Here $U$ is a characteristic speed, $L$ a characteristic length, and $v$ is the kinematic viscosity. Airflow over complex structures will produce a local turning of the flow, or regions of vortical flow, as will the requirement that the velocity of the air become zero on the solid surface itself. When the fluid moves at a high enough speed (or the viscosity is low enough) the inertial forces in the flow dominate the viscous forces and disturbances such as those caused by irregularities in the solid surface grow and eventually cause the fluid to move with a disorganized, turbulent motion. Because turbulent flows involve fluid structures which span many orders of magnitude in size, approximate treatments of turbulence are required in largescale fluid dynamics calculations such as those used here. In this case the effect of the turbulent motion was modeled as an additive term to the overall fluid viscosity by using a parameter called the turbulent or eddy viscosity. The value for this effective turbulent viscosity was obtained via a two-parameter approximation known as the $k-\epsilon$ model. If $k$ is the turbulent kinetic energy per unit mass and if $\epsilon$ is the turbulent energy dissipation rate (ultimately into heat) per unit mass, then an effective turbulent viscosity $v_{t}$ can be written as

$$
v_{t}=C k^{2} / \epsilon,
$$


where $C$ is a constant. Further details about the nature of this approximation are contained in the Appendix. This approximation is widely used and has been found to be in good agreement with experimental data (Schlichting 1979).

The boundary conditions in all cases are the following: the velocity component aligned with the incoming air flow direction is set equal to the free-stream value on the inlet, sides and top of the flow domain while all other components on these faces were set to zero. The inlet boundary value is placed sufficiently upstream from the area of interest so that the flow in those areas reflects the effects of the upstream topography and not the boundary condition. This criterion simply requires that the boundary layer at the surface be well established before any topological features of interest interact with the flow, and it is easily met. The same is true for the boundaries located at the sides and top of the flow domain. All velocity components on solid surfaces are set to zero. A stress-free boundary condition is imposed upon the downstream boundary that allows the air to exit the flow domain in a smooth and unconstrained manner.

The solution of the hydrodynamic equations is carried out through the finite element method (e.g., Taylor \& Hughes 1981). The nonlinear nature of the fluid equations at high Reynolds numbers requires that the system of equations be solved by an iterative method. The combination of the large scale of the problem and the need for high spatial resolution implies that only state-of-the-art computing facilities can address this class of problem. Accurate modeling of the turbulent boundary layer makes the demand on computing capability especially critical, since the computational mesh must be extremely fine near the surface of the ground. In a simulation covering an area of 1.75 square miles, the smallest mesh separation next to the surface is approximately four inches in height. The calculations and post processing were carried out at the San Diego Supercomputer Center where the Cray Y-MP and Cray C90 computers were used. Each simulation required approximately $5 \mathrm{~h}$ of CPU time and used about $16 \mathrm{MW}$ of memory.

\section{RESULTS}

\subsection{General Considerations}

The flow diagnostics available from the simulations are numerous and comprehensive; however, for site evaluation in terms of seeing the principal variables used are the velocity field and the vorticity, $\omega=\nabla \times v$. The velocity field provides a direct measure of the effect of significant topographical features, for example, the formation of vortices downstream of mountain ridges, and it also indicates the presence of shear and deflections in the fluid flow. The vorticity provides a measure of the mixing of the flow introduced by the topographical features. Since the main flow in the far field is uniform, the vorticity is a very valuable measure of the extent of the boundary layer created by the air as it flows over the surface of the site. An additional diagnostic is the pressure field, which can be used as a consistency check on the other flow variables and to detect any anomalies introduced by poorly posed initial or boundary conditions.

FIG. 6. velocity field in a vertical plane passing through the Mauna Kea site for the large scale simulation with a free stream wind speed of $20 \mathrm{~m} / \mathrm{s}$ from the west. The site location on the summit ridge is denoted by the letter A; the upstream topological feature of concern is labeled by the letter $B$. The inset shows a close up view of the flow in 
the region upstream of the summit, which is the left most peak in the inset. Note that even at this high speed the vortex produced by the upstream peak "closes" before it reaches the site on the summit ridge.

Fig. 8. Velocity field in a horizontal plane for the large-scale Mauna Kea simulation with a westerly wind at 20 $\mathrm{m} / \mathrm{s}$. The plane passes below the summit ridge, where no vectors are shown, and just above the small peak upstream of the summit ridge. Disturbed flow due to the upstream feature can clearly be seen. North is at the top.

In general the diagnostics shown here will provide velocity information in the form of a velocity field in a given plane-e.g., the value of velocity vectors in an $x-y$ plane. Vorticity information is displayed as the absolute magnitude of the vorticity vector, $|\omega|=\left[\omega_{x}^{2}+\omega_{y}^{2}+\omega_{z}^{2}\right]^{1 / 2}$, in a given plane or slice through the computational volume. An enormous amount of data is available from three-dimensional calculations such as those described here. The results given in the figures are a very small subset of the data examined in order to arrive at the conclusions drawn from these simulations.

\subsection{Mauna Kea}

\subsubsection{Large-scale simulation}

As described in Sec. 2, the purpose of this series of simulations was to determine the effects of large-scale topographical features upwind of the proposed site on the summit ridge. Of particular concern was the effect of the prominent conical feature lying to the west and slightly south of the proposed site. This feature, which can be seen in Fig. 1 and Fig. 2, has an associated ridge which lies directly west of the site. Figure 6 shows the velocity field in a vertical plane passing through the site for a westerly wind of $20 \mathrm{~m} / \mathrm{s}$. The downstream vortex produced by features described above can be clearly seen; however, it can also be seen that the vortex "closes" before the summit ridge and that the flow over the summit is smooth and undisturbed under these conditions. As expected, wind speeds of less than $20 \mathrm{~m} / \mathrm{s}$ produced vortex closure even further upstream from the summit ridge. Because parcels of air at differing temperatures lying along the light path cause the degradation in seeing, closure of the vortex upstream of the site will not by itself assure good conditions unless volume elements of air at different temperatures entrained in the vortex are also completely mixed into homogeneous flow by the time the airflow passes over the site. That this is the case can be seen by invoking the "Reynolds analogy," which entails comparison of the thermal diffusion and the momentum diffusion in the flow. The ratio of the momentum diffusivity (kinematic viscosity) to the thermal diffusivity is the Prandtl number Pr. The Reynolds analogy states that if this ratio is one or less then the scales of damping or diffusion of turbulence and temperature in homogeneities are essentially the same. In this case the turbulent Prandtl number Pr, $t$ should be used which includes the total (eddy and molecular) viscosity. For air under these conditions $\operatorname{Pr} \approx 0.78$ and $\operatorname{Pr}, t=0.86-0.90$ (e.g., Kays \& Crawford 1980), hence the regions which bound the vorticity of the flow also enclose the regions of thermal inhomogeneity). Figure 6 also shows the turbulent vortex produced by the summit ridge itself, indicating that sites to the east of the summit would experience degraded seeing under these wind conditions. Figure 7 (Plate 192) shows the values of the vorticity in the same plane as Fig. 6. This figure clearly shows the presence of the turbulent boundary layer next to the surface as well as the turbulence induced in the flow by prominent topographic features. 
FIG. 10. High-resolution calculation of velocity vectors for an easterly wind of II $\mathrm{m} / \mathrm{s}$ at the proposed Mauna Kea site. View is from the northeast, in contrast to that of Fig. 9.

A similar result occurs for winds from the east. Here the smooth and rapidly dropping terrain to the east of the summit provides laminar flow over the summit at all wind speeds. However, immediately west and below the summit the velocity field is characterized by considerable vorticity. Some consequences of this flow will be discussed in Sec. 3.2.2. Figure 8 shows the velocity field in a horizontal plane which passes just below the summit ridge but just above the conical features lying west of the summit. For westerly winds Fig. 8 clearly shows the disruption caused by the upstream topography as well as the complex flow around and behind the summit ridge. More importantly, Fig. 8 again shows that the effects of the upstream topography do not propagate to the site location on the summit ridge. This figure also shows that even for winds from the west-southwest it is unlikely that flow at the site would be disturbed by upstream features. (Experience has also shown that southwest winds on Mauna Kea are often accompanied by cloudy conditions.) Hence the large-scale Mauna Kea simulations show that the principal topographic features on the summit region do not result in disturbed airflow over the proposed site under the range of wind conditions normally expected during clear weather.

Fig. 13. Comparison of the numerical simulations with empirical data. The left panel shows the run of velocity in a vertical plane at the primary Cerro Pachon site from the simulations. The length of the arrows at the top correspond to the free wind speed of $10 \mathrm{~m} / \mathrm{s}$. The right panel shows velocity data on the same scale obtained from a test tower at the primary Cerro Pachon site when the free wind speed was $\sim 10 \mathrm{~m} / \mathrm{s}$ from the north. The agreement between the data and the numerical simulation, particularly in the shape of the velocity profiles, is very good.

\subsubsection{Small-scale simulations}

The purpose of these calculations is to determine the detailed character of the turbulent boundary layer around the telescope enclosure and its immediate environment as a function of wind direction and speed. The site location and enclosure used in the set of simulations is shown in Fig. 3, and again both easterly and westerly winds were simulated with wind speeds ranging from 2 to $20 \mathrm{~m} / \mathrm{s}$. Figure 9(a) (Plate 193) is a plot of the magnitude of the vorticity in a vertical plane at the site for a west wind at $11 \mathrm{~m} / \mathrm{s}$. This wind speed is the 70th percentile speed at the site; i.e., $70 \%$ of the time the wind is at or less than this speed. Figure 9(a) again shows the effect of the boundary layer on the surface. The key result shown in this figure is the height of the surface boundary layer relative to the height of the model enclosure. The enclosure rises $38.5 \mathrm{~m}$ above the terrain, while the figure shows the boundary layer at the site is much less than one half this value. Hence the proposed height of $20 \mathrm{~m}$ for the support pier of the telescope will place the light path above the turbulent boundary layer for almost all observing conditions. The boundary layer becomes somewhat thicker as the wind speed decreases, but in all cases simulated (2-20 $\mathrm{m} / \mathrm{s}$ ) the thickness of the boundary layer remained below the $20 \mathrm{~m}$ pier height of the telescope. The vorticity at the site for an easterly wind of $11 \mathrm{~m} / \mathrm{s}$ is shown in Fig. 9(b) (Plate 193), and Fig. 10 shows the velocity vectors for an east wind at $11 \mathrm{~m} / \mathrm{s}$. Again, the boundary layer at the enclosure lies well below the proposed pier height for the telescope, which shows that both the site and the telescope enclosure are satisfactory for easterly winds. It is important to note that the velocity vectors shown in Fig. 10 are the full threedimensional velocity vectors and not just the velocity components lying in that plane. Hence the direction of the vectors near the structure is a 
reflection of the turning of the flow as it is decelerated and moves around the cylindrical structure. Figures 9(b) and 10 also agree with what is widely known by experienced observers at Mauna Kea, namely, that when winds are from the east, conditions are more calm at the Infrared Telescope Facility (IRTF) than at the 88 in. telescope. The former facility lies west of the summit ridge below the access road to the summit, whereas the latter is on the summit ridge. The figures show a much lower wind velocity below and to the west of the ridge, in agreement with what is observed on the mountain. Figures 9 and 10 clearly show that the downstream influence of structures as large as an $8 \mathrm{~m}$ telescope enclosure is of considerable extent. Given the proximity of observing facilities on the summit ridge of Mauna Kea, the issue of degradation of seeing due to airflow over and around adjacent structures naturally arises. Fortunately, this ridge is oriented north-south, while the prevailing winds are east-west. Under these conditions the region of disturbed airflow around a structure extends outward in a direction perpendicular to the airflow to a distance roughly half the structure diameter. The spacing of observing facilities on the ridge is large enough that this north-south region of turbulent air causes negligible degradation of seeing at adjacent facilities. In the case of winds from the north or south, considerable interference will be present because adjacent facilities lie in each other's turbulent wake. However, such wind conditions are rare at this site, and when they do occur they are often accompanied by significant cloud cover.

\subsection{Cerro Pachon}

The objective here was to compare conditions at the pfimary and secondary sites shown in Fig. 5 under wind conditions of about $10 \mathrm{~m} / \mathrm{s}$ from the north. Figure 11 (Plate 194) shows the vorticity in vertical planes passing through the two sites; this large scale view gives the general impression that the boundary layer characteristics are similar at the two locations. This impression is confirmed in Figs. 12(a) and 12(b) (Plate 195), which is a detailed close-up view of the vorticity at each site. This comparison shows that there are no major differences in the height or the intensity of the boundary layer between the primary and secondary sites. Plots of the velocity vectors in the horizontal and vertical planes at the two locations also confirm this conclusion. As a result, the primary site was chosen for the location of the $8 \mathrm{~m}$ telescope because of significant savings in site preparation costs and construction costs made possible by use of this site.

Figure 13 summarizes a check on the accuracy of the simulations. This figure presents a closeup of the velocity vectors in a vertical plane at the primary site from the simulations, together with a plot of data from the test tower located at the site for the same vertical scale and comparable free stream wind speed. The similarity in the velocity profiles between the simulations and the test data provides an encouraging confirmation of the accuracy of the simulations.

\section{CONCLUSIONS}

The current generation of supercomputers has made possible the calculation of fluid flow in three dimensions around complex geometries. This computational capability has been used to provide realistic evaluations of prospective sites for the $8 \mathrm{~m}$ telescopes being constructed by the 
Gemini consortium in a manner that could not be economically achieved by other means. The simulations have confirmed the laminar nature of the flow over the Mauna Kea site in spite of major topographic features upstream; they have determined that the turbulent boundary layer will not intercept the light path if a telescope pier height of $20 \mathrm{~m}$ is used, and they have enabled an informed choice to be made between two possible sites in the southern hemisphere.

The value of employing such simulations for similar projects and for other aspects of the Gemini project is now clear. Evaluation of pressure distributions across mirrors and their support systems under various wind conditions, simulation of airflow within proposed telescope enclosures, the effects on "mirror seeing" of temperature differentials between the mirror and the ambient air, and the effects on "dome seeing" of modifications to existing telescope enclosures are all problems that can be treated with this technique. Some of these problems are currently being investigated, and it is clear that additional applications will be found in the near future.

\section{APPENDIX A: EQUATIONS OF MOTION}

The equations of motion that describe the air flow modeled in this effort are given by

$$
\begin{aligned}
& \nabla \cdot \mathrm{v}=0 \\
& (\mathrm{v} \cdot \nabla) \mathrm{v}=\frac{-1}{\rho} \nabla \rho+v \nabla^{2} \mathrm{v}
\end{aligned}
$$

The air velocity vector is represented by $\mathbf{v}$, the air pressure is $\mathrm{p}$, and the kinematic viscosity of the air is $v$. A point to note in the second equation is that the convective term on the left is balanced on the right by a source term (the pressure gradient) and the viscous dissipative or diffusive term. For low speed or highly viscous flows where the convective term can be neglected the system is elliptical in nature and the resultant Poisson's equation can easily be solved. For high-speed flows or fluid media with low viscosity, such as in this case, the equations are highly nonlinear in nature and an iterative or other numerical approach is required.

The regions of the flow domain that are of the greatest interest are those where there is a transition from the freestream conditions to those imposed upon the flow by the surface. This transition region or boundary layer is usually the region where momentum and heat are transferred into or out of the flow from the boundaries. An overview of these processes can be found in Schlichting (1979), and a practical treatment of modeling concerns, such as why the isothermal assumption used in this effort is valid, can be found in Cebeci \& Bradshaw (1988).

An important aspect of the flow is indicated by the value of the Reynolds number. Laminar flow is an orderly regular flow of the fluid that moves in regular layers, or lamina, at characteristically low Reynolds numbers. As the Reynolds number of the flow increases (typically by increasing the flow speed or the length scale), the nonlinear terms in the equations begin to dominate, and disturbances or asymmetries present in the flow are amplified, causing the flow to be broken up into disorganized and chaotic turbulent pieces. The following turbulence model was used to treat this aspect of the flow. 


\section{APPENDIX B: TURBULENCE MODELING}

The flow turbulence in this study was modeled using a two-equation model known as the $k$ $\epsilon$ technique. The $k-\epsilon$ model involves modeling the turbulent kinetic energy $k$, and the turbulent energy dissipation rate $\epsilon$, which is the rate of conversion of turbulent energy to thermal energy by the diffusive effects of the fluid's molecular viscosity. These quantities are treated as additional independent flow variables.

To develop this model, the momentum equation is modified via perturbation analysis to be

$$
(\overline{\mathrm{v}} \cdot \nabla) \overline{\mathrm{v}}=\frac{-1}{\rho} \nabla \bar{\rho}+v \nabla^{2} \overline{\mathrm{v}}-\nabla \cdot \mathrm{v}^{\prime} \mathrm{v}^{\prime}
$$

where terms denoted by (-) are mean quantities and those denoted by (') are perturbed quantities. The term $\mathbf{v}^{\prime} \mathbf{v}$ ' is a form of the Reynolds stress tensor. In more complicated turbulence models this quantity is modeled directly. In the $k-\epsilon$ model it is approximated as a function of the gradient of the mean flow velocity and the turbulent kinetic energy.

For laminar flows the viscosity in the flow equations is assumed to be the molecular viscosity, $v_{0}$, but for turbulent flows the viscosity used is taken to be the sum of the molecular viscosity and an eddy viscosity, $v_{t}$, and given by

$$
v=v_{0}+v_{t}
$$

The parameters $\mathrm{k}$ and $\epsilon$ are modeled as independent flow variables at each point with each subject to a governing differential equation. The turbulent eddy viscosity is then found via the relationship,

$$
v_{t}=C_{\mu} k^{2} / \epsilon
$$

where the coefficient $C_{\mu}$ is an empirically determined constant. Additional details of turbulence modeling can be found in Wilcox (1993).

\section{APPENDIX C: NUMERICAL TECHNIQUE AND SOFTWARE}

The specific numerical technique used here is a fluid dynamic implementation of the finite element method (FEM). The software employed is a commercial package produced by Fluid Dynamics International, Inc. and is named FIDAP. In the FEM technique discrete nodes are distributed throughout the flow domain, and the nodes are organized into volume elements with associated edges and surfaces. Nodes that lie on the boundary of an element may be common to one or more elements and will thus have multiple contributions to the discrete system of equations. The flow variables become individual degrees of freedom at the nodal locations. The heart of the FEM technique lies in the process of going through the list of elements, and, for each element, forming a local matrix that contains the contributions of all of the interpolated values at 
the computational nodes in that element. This local matrix is inserted into a grand, or global, matrix that characterizes the response of the entire flow system to the externally imposed force, flow, or thermal conditions.

Due to the overall unsymmetric nature of the matrix formed by the response of the fluid system, the system cannot be solved directly for the degrees of freedom but must be solved by using an iterative technique. At each iteration, a measure of the global error, or residual, is calculated. The iterative solution process is repeated until some appropriate measure of this residual is below a specified value. A presentation of the general topic and the mathematical basis of FEM can be found in Hughes (1987); a treatment of concerns particular to modeling fluid dynamics problems can be found in Baker (1983), and a presentation of the step-by-step application of the method to two-dimensional flow cases can be found in Taylor \& Hughes (1981).

\section{APPENDIX D: FIDAP}

FIDAP is a general purpose FEM analysis code consisting of modules that allow the user to set up the flow simulation with the pre-processing utilities, to run the flow solving modules for a specified number of iterations, to restart the calculation if the solution has not converged sufficiently during the previous attempt, and to use the post-processing module to view the results of the calculations. For example, the large-scale calculation over Mauna Kea set the dimensions of the flow domain to 81 by 49 by 21 , giving 83,349 elements. Of this number 3,840 were special four-node wall elements necessary to model the turbulent flow directly adjacent to the solid boundary of the mountain surface and 76,800 were general eight-node brick elements used in the bulk of the flow domain.

The nonlinear, three-dimensional turbulent equations were supplemented by the following conditions: the flow was incompressible, steady state, and isothermal; the fluid was given a Newtonian viscosity with only one gas species in a single phase; the flow domain had fixed surface boundaries where the momentum equation was explicitly modeled; and the formulation of the pressure term was "mixed" where the pressure term was explicitly discretized as a degree of freedom.

The actual solution of the matrix equation defined by the system of flow equations was accomplished by the use of a "segregated" solver which, instead of assembling a global matrix containing all of the contributions from all of the degrees of freedom, forms submatrices that handle a subset of the flow equations. The submatrices contain additional terms that allow each submatrix to be related to the others. The advantage of using this technique is that it allows use of a global matrix which is too large to fit into the core memory at one time.

Boundary values of a uniform incoming velocity profile were specified at the inlet, top, and sides of the flow domain; zero velocity components were specified on the solid surfaces, and stress-free outflow conditions were imposed on the downstream boundary of the flow domain. Turbulence parameters were specified through the boundary values of $k$ and $\epsilon$. 


\section{REFERENCES}

- Baker, A. S. 1983, Finite Element Computational Fluid Mechanics (McGraw-Hill, New York), pp. 233-305, pp. 421-470

- Cebeci, T. \& Bradshaw, P. 1988, Physical and Computational Aspects of Convective Heat Transfer (Springer, New York), pp. 1-40

- Hughes, T. J. 1987, The Finite Element Method (Prentice Hall, Princeton), pp. I- 184

- Kays, W. M., \& Crawford, M. E. 1980, Convective Heat and Mass Transfer (McGraw-Hill, New York)

- Mountain, M., Kurz, R., \& Oschmann, J. 1994, SPIE Proc., 2199, 41

- Raybould, K., Gillett, P., Hatton, P., Pentland, G., Sheehan, M., \& Warner, M. 1994, SPIE Proc., 2199, 376

- Schlichting, H. 1979, Boundary Layer Theory (McGraw-Hill, New York), pp. 5-82, pp. 408595

- Taylor, C., \& Hughes, T. G. 1981, Finite Element Programming of the Navier-Stakes Equations (Pineridge Press, Swansea, UK), pp. 1-58

- Wilcox, D. C. 1983, Turbulence Modeling for CFD (DCW Industries, La Canada, CA), pp. I- 170

- Stepp, L. 1994, in Advanced Technology Optical Telescopes V, edited by L. Stepp, SPIE Proc. 2199 (SPIE, Bellingham)

- Wong, W.-Y., \& Forbes, F. F. 1991, SPIE Proc., 1532, 15 